\title{
Prospección química del bosque de galería del río Uruguay
}

\author{
Ana Bertucci, ${ }^{1}$ Federico Haretche, ${ }^{2}$ Cristina Olivaro, ${ }^{1}$ Alvaro Vázquez ${ }^{*, 1}$ \\ ${ }^{I}$ Cátedra de Farmacognosia y Productos Naturales, Departamento de Química Orgánica, Facultad de Química, \\ CC 1157, Montevideo 11800, Uruguay, \\ ${ }^{2}$ Museo y Jardín Botánico “Atilio Lombardo" 19 de Abril 1181, Montevideo 11700, Uruguay
}

\begin{abstract}
RESUMO: "Triagem fitoquímica de plantas nativas do rio Uruguai". Prospecção da biodiversidade é a exploração de um ecossistema para se obter informações sobre possíveis utilizações inovadoras em diferentes processos de produção humanos. Exemplos do tipo de informação pretendida são os dados científicos contidos no material genético dos organismos vivos, os compostos químicos produzidos por eles e suas interações ou no conhecimento das pessoas que já interagiram com eles de várias maneiras. Uma das ferramentas de prospecção da biodiversidade é a procura por informações químicas. Esta informação está contida nos compostos produzidos por muitos seres vivos para comunicar-se, defender-se e trocar informações, e eles são conhecidos como metabólitos secundários. Neste projeto, nós propomos a prospecção botânica, química e biológica da biodiversidade da mata ciliar da Bacia do Rio Uruguay próxima ao riacho Guaviyú. Este ecossistema particular apresenta uma substancial biodiversidade de aproximadamente 200 espécies com um perfil subtropical incomum.
\end{abstract}

Unitermos: Bioprospeção, triagem fitoquímica.

\begin{abstract}
Phytochemical screening of the native forest of Uruguay River". Biodiversity prospecting is the exploration of an ecosystem for information regarding possible innovative uses in different human production processes. Examples of the kind of information sought are the scientific data contained in the genetic material of living organisms, the chemical compounds they produce and their interactions or in the knowledge of people that have interacted with them in various ways. One of the tools of biodiversity prospecting is searching for chemical information. This information is contained in the compounds produced by many living beings to communicate, to defend themselves and exchange information, and they are known as secondary metabolites. In this project, we propose the botanical, chemical and biological biodiversity prospecting of the gallery forest of the Uruguay River Basin near the Guaviyú stream. This particular ecosystem presents a substantial biodiversity of approximately 200 species with an unusual subtropical profile.
\end{abstract}

Keywords: Bioprospection, phytochemical screening.

\section{INTRODUCCIÓN}

Las especies arbóreas que forman parte de las comunidades nativas del Uruguay son alrededor de 170 ascendiendo a unas de 250 si se consideran también las especies arbustivas y caméfitas sobre un total algo mayor a las 2600 especies de plantas vasculares que crecen en el país. Este es un número considerablemente más alto al de la provincia de Buenos Aires (Argentina) a la que tradicionalmente se asocia en la bibliografía a la flora uruguaya, ubicándolas en la misma Provincia fitogeográfica (Escudero, 2004). La vegetación leñosa, arbóreo arborescente del Uruguay incluyendo a los palmares, según la carta forestal (Uruguay, 1980) cubre el $3,7 \%$ de la superficie del territorio nacional, totalizando 667.315 hás. Dichas agrupaciones boscosas ocupan diferentes ambientes que determinan situaciones en la mayoría de los casos perfectamente diferenciables entre sí desde un punto de vista ecológico geográfico, lo que no ocurre si sólo se tiene en cuenta la localización geográfica de las especies leñosas.

Atendiendo a su fisonomía y a las características fisiográficas del área que ocupan, se han diferenciado seis tipos de formaciones boscosas (Chebataroff, 1942; Grela, 2004): Bosque de galería, fluvial o ribereño (en las márgenes de los cursos de agua); Bosque de parque (en zonas próximas al litoral del Río Uruguay, como nexo entre el bosque fluvial y las comunidades herbáceas); Bosque de quebrada (en las quebradas húmedas y valles escarpados del norte y noreste del país); Bosque serrano (en las serranías y "mares de piedra"); Bosque costero (en las costas del Río de la Plata y Océano Atlántico); y Palmares (agrupaciones prácticamente puras de palmeras).

En las costas e islas del río Uruguay (departamentos del norte: Artigas, Salto y Paysandú) y en los afluentes (ríos Arapey, Queguay, etc.) la vegetación del bosque de galería adquiere un "aspecto selvático" 
con especies propias de regiones subtropicales tales como la caña tacuaruzú (Guadua angustifolia) de 10-12 $\mathrm{m}$ de altura y diámetro de $10-12 \mathrm{~cm}$. A este fenómeno se le denomina intrusión de vegetación subtropical. Especies de la Provincia Paranense se desplazan hacia el sur por los ríos Uruguay y Paraná aprovechando el microclima ribereño, llegando hasta Punta Lara (Provincia de Buenos Aires).

En estos tipos de bosques se encuentran 70 especies de árboles y 57 de arbustos $(50 \%$ de la flora arbórea y arbustiva del Uruguay) y 78 de enredaderas (88\% del total).

En los últimos años, la pérdida de especies y hábitats enteros ha ocurrido a un ritmo sin precedentes. La extinción de cada especie adicional lleva a la pérdida irreversible de genes únicos, que podrían relacionarse con el desarrollo de alimentos o medicamentos. Al mismo tiempo el auge del uso de los productos naturales en el área de la salud como productos farmacéuticos, nutracéuticos o cosméticos abre una esperanza para su uso. La bioprospección hace posible la conservación y el desarrollo económico a través de la explotación sustentable de la biodiversidad (Albuquerque \& Hanazaki, 2006; Artuso, 2002; Chapela, 1994; Bell, 1993).

Una de las formas de bioprospección es la búsqueda de información química, contenida en los compuestos producidos por muchos seres vivos como medios de comunicación, defensa, e intercambio de información, y conocidos como metabolitos secundarios. Los metabolitos secundarios de cada especie son extraordinariamente diversos, y la información contenida en sus estructuras es de especial interés para la industria farmacéutica, pues dichos compuestos han sido hasta ahora la fuente principal de innovación en el descubrimiento de nuevas medicinas (Barbosa-Filho et al., 2007; Saúde-Guimarães et al., 2007; Rocha et al., 2007; Amaral et al., 2006; Barbosa-Filho et al., 2006a,b,c; Balick \& Mendelsohn, 2000; Grifo, 1996; McChesney, 1996). En los países desarrollados alrededor del $25 \%$ de las prescripciones dispensadas son drogas derivadas de productos naturales. En los EEUU y UK 119 compuestos derivados de 90 familias vegetales son considerados drogas de primer orden, incluyendo entre ellas 12 de las 25 más vendidas. Aunque los productos naturales comprenden solo $1 \%$ de las estructuras químicas publicadas representan el 3.5\% del mercado farmacéutico de mas de U\$S 250 millardos (Nisbet \& Moore, 1996).

En este trabajo se presenta los resultados de la prospección química de 26 especies nativas encontradas en el bosque de galería del río Uruguay.

\section{MATERIALES Y MÉTODOS}

\section{Selección del área de estudio}

El relevamiento se realizó eligiendo parcelas representativas dentro de la zona en estudio, dos áreas linderas al Río Uruguay, una sobre el Arroyo Guaviyú y otro en la zona de canteras existentes en la zona.

\section{Colecta y estudio botánico}

El relevamiento se desarrolló a través de transectas perpendiculares al Río Uruguay ubicadas alrededor del punto $31^{\circ} 43^{\prime} 30.00^{\prime \prime} S, 58^{\circ} 01^{\prime \prime} 43.31^{\prime \prime} O$ y que abarcaron toda la profundidad del monte ribereño hasta la zona de transición y herbácea posterior. Se tomaron puntos sobre esas transectas y fueron relevados todos los ejemplares comprendidos en un radio de $4 \mathrm{~m} \mathrm{del}$ punto de selección. Los ejemplares fueron identificados, medidos (altura y circunferencia a $120 \mathrm{~cm}$ de la base) y se tomaron muestras de herbario. De aquellas especies que por datos etnobotánicos o etnofarmacológicos previos se esperaba fueran interesantes, se realizaron colectas a fines de análisis.

\section{Selección de muestras para estudio}

Las muestras para estudio fueron seleccionadas tomando en cuenta los criterios previamente definidos y algunos otros aspectos como tipo de vegetación o abundancia.

\section{Preparación de extractos y screening fitoquímico}

Se realizaron extractos en $\mathrm{CHCl}_{3}$, acetona y $\mathrm{EtOH} / \mathrm{H}_{2} \mathrm{O}$ 70:30 de forma de abarcar el mayor rango de polaridades. El screening fitoquímico se realizó por cromatografía en capa fina (TLC), utilizando solventes de corrida y reveladores específicos para cada grupo de compuestos (Marini-Bettolo et al., 1981).

\section{RESULTADOS Y CONCLUSIONES}

Las plantas colectadas fueron 26, pertenecientes a 15 familias botánicas diferentes, cubriendo de esta manera una amplia biodiversidad. Los datos etnofarmacológicos de las mismas se detallan en la Tabla1.

Se realizaron en total 66 extractos cuyo cribaje fitoquímico se reporta en la Tabla 2. En resumen 22 especies dieron un resultado positivo para esteroides $\mathrm{y} / \mathrm{o}$ triterpenos, todas fueron positivas para terpenos menores, 13 para taninos, 12 para alcaloides, 18 para saponinas, 24 para flavonoides, 20 para otros glicósidos fenólicos. Solo una especie, Paulinia ellegans, dio un resultado positivo para cardenólidos.

\section{AGRADECIMIENTOS}

Los autores agradecen el apoyo del Programa de Desarrollo Tecnológico (PDT) a través del proyecto $32 / 34$. 
Tabla 1. Datos etnobotánicos de las especies identificadas en el relevamiento.

\begin{tabular}{|c|c|c|}
\hline Nombre científico & Nombre común & Uso tradicional \\
\hline Combretum fruticosum & Flor de cepillos & Ornamental. \\
\hline Croton tenuissimus & & $\begin{array}{l}\text { El látex de otras plantas del género se emplea como cicatrizante, para } \\
\text { aliviar picaduras de insectos y en casos de aftas y llagas bucales } 1 \text {. }\end{array}$ \\
\hline Eugenia mansoni & Pitanga amarga & Idem. \\
\hline Eugenia uniflora & Pitanga & $\begin{array}{l}\text { Cocimiento de frutos como tónico. Infusión de hojas como febrífugo. } \\
\text { Infusión de las hojas al } 1 \% \text { se emplea como estomacal y antidiarreica. } \\
\text { El licor producido por la maceración de frutos maduros en caña con } \\
\text { azúcar es estomacal } 4,8,6,1,15 \text {. }\end{array}$ \\
\hline Eugenia repanda & Ñangapiri negro & Idem. \\
\hline Galianthe brasiliensis & Poaia-do-campo & Emetico 8. \\
\hline Guettarda uruguensis & Jazmín del Uruguay & Madera, ornamental 6. \\
\hline Hexachlamys edulis & Ubajay & Dulces de frutos 4. \\
\hline Inga uruguensis & Inga & $\begin{array}{l}\text { Cocimiento de corteza como colorante. Tambien como hemostàtico. } \\
\text { La resina del fruto se aplica como antiodontálgica } 1,9,15 \text {. }\end{array}$ \\
\hline Luehea divaricata & Francisco Alvarez & $\begin{array}{l}\text { Infusión de las flores al } 1 \% \text { como sedante. Infusión de hojas como } \\
\text { antiinflamatorio. } \\
\text { Decocción de la corteza como antidiarreica y digestiva, y en forma de } \\
\text { baños vaginales y hemostático en heridas de piel } 1,3,4,5,15 \text {. }\end{array}$ \\
\hline $\begin{array}{l}\text { Mitracarpus } \\
\text { megapotamicus }\end{array}$ & Peladilla & Antimalárico 17. \\
\hline Myrcianthes cisplatensis & Guayabo colorado & Madera. \\
\hline Nectandra angustifolia & Laurel mini & Tratamiento de reumatismo, artritis y dolor. \\
\hline Paullinia elegans & Cipó-timbó & Sin datos. \\
\hline Pavonia sepium & Malva del bosque & Sin datos. \\
\hline Petunia sp & Petunia & \\
\hline Phyllanthus sellowianus & Sarandí Blanco & $\begin{array}{l}\text { Se emplea la infusión o decocción de partes de la planta como } \\
\text { hipoglicemiante, purgante, diurético, antiasmático. Uso externo como } \\
\text { antiséptico } 1,3,4,6,11,15 \text {. }\end{array}$ \\
\hline Pouteria salicifolia & Mata ojo & Extracto de hojas como vulnerario. Madera 15. \\
\hline Psychotria carthagenensis & $\begin{array}{l}\text { Naranjillo, chal-chal de la } \\
\text { gallina. }\end{array}$ & Alucinógeno 7. \\
\hline Ruprechtia laxiflora & Viraro crespo & Carpintería 6. \\
\hline Ruprechtia salicifolia & Viraró & Madera 6. \\
\hline Terminalia australis & Palo amarillo & Madera. Cocimiento de hojas como astringente $6,15,16$. \\
\hline Teucrium vesicarium & -- & Sin datos. \\
\hline
\end{tabular}

1 Alonso J. y Desmarchelier C. Plantas autóctonas medicinales de la Argentina. Ed. LOLA, 2005. 2 Alonso Paz E. et al. Yuyos Uso racional de las plantas medicinales. Ed.Fin de Siglo, Montevideo, 1992. 3 Arrillaga de Maffei, B. Plantas medicinales. Montevideo: Nuestra Tierra, 1969. 60 p: Nuestra tierra. 4 Arrillaga de Maffei, B. Plantas usadas en medicina natural. -- Montevideo: Hemisferio Sur, 1997. 152 p. 5 Cabrera, A. L. \& E. M. Zardini.. Manual de la Flora de los Alrededores de Buenos Aires. 2a ed. Acme, Buenos Aires. 1978, 755 pp. 6 Chifa C. y Ricciardi A. Plantas de uso en Medicina Vernácula del centro del Chaco Argentino. Fundaciòn Miguel Lillo, Tucumán, 2001. 7 Copetti, V. Tratado teórico-práctico de Farmacognosia, con una relación circunstanciada sobre las plantas medicinales comunes del Uruguay, Monteverde, Montevideo, 1918. 8 De Lucca D., M. y Zalles A., J. Flora medicinal Boliviana. Ed. Los amigos del libro, Bolivia, 1992. 9 Flora de la región de Salto Grande http://www.oni.escuelas.edu.ar/2002/ entre_rios/un-gran-salto/paginas/todoslosframes.htm. 10 Goyeneche, B.; Diccionario de medicina rural ó sea, propiedades medicinales de las plantas del país - Paysandú ,s/f. 11 Gupta M., 270 Plantas Medicinales Iberoamericanas. CYTED, Subprograma X, Colombia, 1995. 12 Jozami, J. M. y J. de D. Muñoz.. Arboles y Arbustos Indígenas de Entre Ríos. 3a. ed. IPNAYS CONICET. 1983, Santa Fe. 13 Lombardo, Atilio, Gonzalez, Matías - Coppetti, Víctor -; Plantae Diaphoricae: Florae Uruguayensis (Tomo I) Montevideo, 1928 14 Lombardo, A., Copetti, V., González, M., y Vallarino A. Plantas de la Medicina Popular del Uruguay, Tall. Graf. Cerrito, Montevideo, 1937. 15 Lahitte, H. B.; Hurrell, M. J. B.; Jankowski, L.; Haloua, P.; Mehltreter, K. "Plantas Medicinales Rioplatenses". Ed. L.O.L.A. (Literature of Latin America). Buenos Aires, Argentina. 1998, 240 pp. 16 Ratera L. y Ratera M. Plantas de la flora Argentina empleadas en la medicina popular. Ed. Hemisferio Sur, Buenos Aires, 1980. 17 Toursarkissian, M. Plantas medicinales de la Argentina : Sus nombres botánicos, vulgares, usos y distribución geográfica. Buenos Aires: Hemisferio Sur, 1980. $178 \mathrm{p}$ 
Tabla 2. Screening fitoquímico de las muestras colectadas.

\begin{tabular}{|c|c|c|c|c|c|c|c|c|c|c|}
\hline Especies & Parte usada & Extracto & Tri/Est & Terp & Tan & Sap & Alc & Fla & Gli & Card \\
\hline \multirow[t]{3}{*}{ Combretum fruticosum } & Hoja & 1 & - & + & + & + & - & + & + & - \\
\hline & & 2 & + & + & - & - & - & + & - & - \\
\hline & & 3 & - & + & - & - & - & - & - & - \\
\hline \multirow[t]{3}{*}{ Croton tenuissimus fem } & Hoja & 1 & + & + & + & + & - & + & + & - \\
\hline & & 2 & + & + & - & - & - & + & - & - \\
\hline & & 3 & + & + & - & - & - & + & - & - \\
\hline \multirow[t]{3}{*}{$\operatorname{masc}$} & & 1 & + & + & + & + & - & + & + & - \\
\hline & & 2 & + & + & - & - & - & + & - & - \\
\hline & & 3 & + & + & - & - & - & + & - & - \\
\hline \multirow[t]{3}{*}{ Eugenia mansoni } & Hoja & 1 & - & + & - & + & - & + & + & - \\
\hline & & 2 & + & + & - & - & + & + & - & - \\
\hline & & 3 & + & + & - & - & + & - & - & - \\
\hline \multirow[t]{3}{*}{ Eugenia repanda } & Hoja & 1 & - & - & + & + & - & + & + & - \\
\hline & & 2 & + & + & - & - & + & + & - & - \\
\hline & & 3 & + & + & - & - & + & + & - & - \\
\hline \multirow[t]{3}{*}{ Eugenia uniflora } & Hoja & 1 & - & - & + & + & - & + & + & - \\
\hline & & 2 & + & + & - & - & - & + & - & - \\
\hline & & 3 & + & + & - & - & + & + & - & - \\
\hline Galianthe brasiliensis & Hoja & 2 & + & + & - & - & - & + & - & - \\
\hline \multirow[t]{3}{*}{ Guettarda uruguensis } & Hoja & 1 & - & - & + & - & - & + & + & - \\
\hline & & 2 & + & + & - & - & - & + & - & - \\
\hline & & 3 & + & + & - & - & + & - & - & - \\
\hline \multirow[t]{3}{*}{ Hexachlamys edulis } & Hoja & 1 & - & - & + & + & - & + & + & - \\
\hline & & 2 & + & + & - & - & - & + & - & - \\
\hline & & 3 & + & + & - & - & + & - & - & - \\
\hline \multirow[t]{3}{*}{ Ingá uruguensis } & Hoja & 1 & + & - & - & - & - & - & - & - \\
\hline & & 2 & + & + & - & - & - & - & - & - \\
\hline & & 3 & + & + & - & - & - & - & - & - \\
\hline Luehea divaricata & Hoja & 1 & + & - & + & + & - & + & + & - \\
\hline & & 2 & + & + & - & - & - & + & - & - \\
\hline & & 3 & + & + & - & - & + & - & - & - \\
\hline $\begin{array}{l}\text { Mitracarpus } \\
\text { megapotamicus }\end{array}$ & Parte aérea & 2 & - & + & - & - & - & + & - & - \\
\hline Myrciantes cisplatensis & Hoja & 1 & - & + & + & + & - & + & + & - \\
\hline & & 2 & - & + & - & - & + & + & - & - \\
\hline & & 3 & - & + & - & - & + & + & - & - \\
\hline Nectandra angustifolia & Hoja & 1 & + & - & - & - & - & + & + & - \\
\hline & & 2 & + & + & - & - & - & + & - & - \\
\hline & & 3 & + & + & - & - & + & - & - & - \\
\hline Paullinia elegans & Hoja & 1 & - & - & - & + & - & + & + & + \\
\hline & & 2 & + & + & - & - & - & + & - & - \\
\hline & & 3 & + & + & - & - & + & - & - & - \\
\hline & Frutos & 1 & - & - & - & - & - & - & + & - \\
\hline Pavonia sepium & Parte aérea & 2 & + & + & - & - & - & - & - & - \\
\hline Petunia sp & Parte aérea & 1 & - & + & - & + & + & + & + & - \\
\hline & & 2 & - & + & - & - & + & + & - & - \\
\hline & & 3 & + & + & - & - & + & - & - & - \\
\hline Phyllanthus sellowianus & Hoja & 1 & - & + & + & + & - & + & + & - \\
\hline & & 2 & + & + & - & - & - & - & - & - \\
\hline & & 3 & + & + & - & - & - & - & - & - \\
\hline Pouteria salicifolia & Hoja & 1 & - & + & - & + & - & + & + & - \\
\hline & & 2 & - & + & - & - & + & + & - & - \\
\hline & & 3 & + & + & - & - & + & - & - & - \\
\hline Psychotria carthagenensis & Hoja & 1 & + & - & + & + & - & + & + & - \\
\hline & & 2 & + & + & - & - & - & + & - & - \\
\hline & & 3 & + & + & - & - & - & - & - & - \\
\hline Ruprechtia laxiflora & Hoja & 1 & - & - & + & + & - & + & + & - \\
\hline & & 2 & + & + & - & - & - & + & - & - \\
\hline & & 3 & + & + & - & - & - & - & - & - \\
\hline Rupretchia salicifolia & Hoja & 1 & - & + & + & + & - & + & + & - \\
\hline & & 2 & + & + & - & - & - & - & - & - \\
\hline & & 3 & + & + & - & - & - & - & - & - \\
\hline Scutia buxifolia & Hoja & 1 & - & - & - & + & - & + & + & - \\
\hline & & 2 & + & + & - & - & - & + & - & - \\
\hline & & 3 & + & + & - & - & - & - & - & - \\
\hline Sebastiana commersoniana & Hoja & 2 & + & + & - & - & - & + & - & - \\
\hline Terminalia australis & Hoja & 1 & - & - & - & + & - & + & + & - \\
\hline & & 2 & + & + & - & - & - & + & - & - \\
\hline & & 3 & + & + & - & - & - & + & - & - \\
\hline Teucrium vesicarium & Hoja & 2 & & & & & & & & \\
\hline
\end{tabular}

Solventes de extracción: 1 EtOH- $\mathrm{H}_{2} \mathrm{O}$ 70:30, $2 \mathrm{CH}_{3} \mathrm{COCH}_{3}, \mathbf{3} \mathrm{CHCl}_{3}$

Clave: Tri/Est (triterpenos/esteroides), Terp (terpenos de bajo peso molecular), Tan (taninos, polifenoles), Sap (saponinas), Alc (alcaloides), Flav (flavonoides), Gli (glicósidos), Card (cardiotónicos). 


\section{REFERENCIAS}

Albuquerque UP, Hanazaki N 2006. As pesquisas etnodirigidas na descoberta de novos fármacos de interesse médico e farmacêutico: fragilidades e pespectivas. Rev Bras Farmacogn 16(Supl.): 678-689.

Amaral FMM, Ribeiro MNS, Barbosa-Filho JM, Reis AS, Nascimento FRF, Macedo RO 2006. Plants and chemical constituents with giardicidal activity. Rev Bras Farmacogn 16(Supl.): 696-720.

Artuso A 2002. Bioprospecting, benefit sharings and biotechnological capacity building. World Development 30: 1335-1368.

Balik MJ, Mendelsohn R 2000. Assessing the economic value of traditional medicines for tropical rainforest. Consev Biol 6: 128-130.

Barbosa-Filho JM, Piuvezam MR, Moura MD, Silva MS, Lima KVB, Cunha EVL, Fechine IM, Takemura OS 2006a. Anti-inflammatory activity of alkaloids: A twenty-century review. Rev Bras Farmacogn 16: 109-139.

Barbosa-Filho JM, Medeiros KCP, Diniz MFFM, Batista LM, Athayde-Filho PF, Silva MS, Cunha EVL, Almeida JRGS, Quintans-Júnior LJ 2006b. Natural products inhibitors of the enzyme acetylcholinesterase. Rev Bras Farmacogn 16: 258-285.

Barbosa-Filho JM, Martins VKM, Rabelo LA, Moura MD, Silva MS, Cunha EVL, Souza MFV, Almeida RN, Medeiros IA 2006c. Natural products inhibitors of the angiotensin converting enzyme (ACE). A review between 1980-2000. Rev Bras Farmacogn 16: 421-446.

Barbosa-Filho JM, Nascimento-Júnior FA, Tomaz ACA, Athayde-Filho PF, Silva MS, Cunha EVL, Souza MFV, Batista LM, Diniz MFFM 2007. Natural products with antileprotic activity. Rev Bras Farmacogn 17: 141-148.

Bell EA 1993. Mankind and plants: the need to conserve biodiversity. Parasitology 106(Suppl.): S47-S53.

Chapela I 1994. Bioprospección, ¿una herramienta para el manejo sostenible de los recursos naturales? Foro Forestal 3: 3-4.

Chebataroff J 1942. La vegetación del Uruguay y sus relaciones fitogeográficas con las del resto de la América del Sur. Revista Geográfica del Instituto Panamericano de Geografía e Historia. México 50-90.

Escudero R 2004. Proyecto Combinado GEF/IBRD “Manejo Integrado de Ecosistemas y Recursos Naturales en Uruguay" Componente "Manejo y Conservación de la Diversidad Biológica" Subcomponente Bosque nativo.

Grela IA 2004. Geografía florística de las especies arbóreas de Uruguay: Propuesta para la delimitación de dendrofloras. Tesis de Maestría en Ciencias Biológicas, Opción Botánica. Programa de Desarrollo de las Ciencias Básicas (PEDECIBA), Ministerio de
Educación y Cultura - Universidad de la República, Montevideo, Uruguay.

Grifo F 1996. Chemical prospecting: an overview of the International Cooperative Biodiversity Group. In Biodiversity, Biotechnology and Sustainable Development in Health and Agriculture.. Washington DC, PAHO: p. 12-26.

Marini-Bettolo GB, Nicoletti M, Patamia M 1981. Plant screening by chemical and chromatographic procedure under field conditions. J Chromatogr 218: 113-217.

McChesney JD 1996. Biological diversity, chemical diversity and the search for new pharmaceuticals. In Balick MJ (ed.) Medicinal resources of the tropical forest. CUP, New Cork, 73-92.

Nisbet L, Moore MM 1997. Will natural products remain an important source of drug research for the future? Curr Opin Biotech 8: 708-712.

Rocha FD, Pereira RC, Kaplan MAC, Teixeira VL 2007. Produtos naturais de algas marinhas e seu potencial antioxidante. Rev Bras Farmacogn 17: 631-639.

Saúde-Guimarães DA, Faria AR 2007. Substâncias da natureza com atividade anti-Trypanosoma cruzi. Rev Bras Farmacogn 17: 455-465.

Uruguay 1980. Ministerio de Ganadería, Agricultura y Pesca. Dirección Forestal Parque y Fauna. Carta Forestal actualizada y suelos de interés forestal. Escala 1: 20.000. Montevideo. s.p 